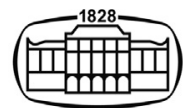

AKADÉMIAI KIADÓ

Journal of Behavioral Addictions

10 (2021) 2, 361-370

DOI:

$10.1556 / 2006.2021 .00032$

(c) 2021 The Author(s)

\section{CASE REPORT}

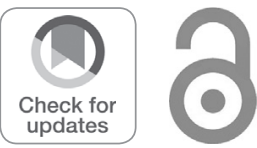

First names were added on 7 April 2022

†These authors equally contributed.

${ }^{*}$ Corresponding author.

E-mail: luigigallimberti.novellafronda@ gmail.com

\title{
The role of repetitive transcranial magnetic stimulation (rTMS) in the treatment of behavioral addictions: Two case reports and review of the literature
}

\section{DIEGO CUPPONE ${ }^{1 \dagger}$, LUIS J. GÓMEZ PÉREZ ${ }^{1 \dagger}$, STEFANO CARDULLO ${ }^{1}$, NICOLA CELLINI ${ }^{2,3,4,5}$, MICHELA SARLO ${ }^{6}$, SILVIA SOLDATESCA ${ }^{1}$, SONIA CHINDAMO ${ }^{1}$, GRAZIELLA MADEO ${ }^{1}$ and LUIGI GALLIMBERTI ${ }^{1 *}$}

\footnotetext{
${ }^{1}$ Novella Fronda Foundation, Piazza Castello, 16 - 35141, Padua, Italy

${ }^{2}$ Department of General Psychology, University of Padua, Padova, Italy

${ }^{3}$ Department of Biomedical Sciences, University of Padova, Padova, Italy

${ }^{4}$ Padova Neuroscience Center, University of Padova, Padova, Italy

${ }^{5}$ Human Inspired Technology Center, University of Padova, Padova, Italy

${ }^{6}$ Department of Communication Sciences, Humanities and International Studies, University of Urbino Carlo Bo, Urbino, Italy
}

Received: November 18, 2020 • Revised manuscript received: February 14, 2021; April 9, 2021; May 5, 2021

- Accepted: May 15, 2021

Published online: July 6, 202

\begin{abstract}
Background: Several behaviors, besides consumption of psychoactive substances, produce short-term reward that may lead to persistent aberrant behavior despite adverse consequences. Growing evidence suggests that these behaviors warrant consideration as nonsubstance or "behavioral" addictions, such as pathological gambling, internet gaming disorder and internet addiction. Case presentation: Here, we report two cases of behavioral addictions (BA), compulsive sexual behavior disorder for online porn use and internet gaming disorder. A 57-years-old male referred a loss of control over his online pornography use, started 15 years before, while a 21-years-old male university student reported an excessive online gaming activity undermining his academic productivity and social life. Both patients underwent a highfrequency repetitive transcranial magnetic stimulation (rTMS) protocol over the left dorsolateral prefrontal cortex (l-DLPFC) in a multidisciplinary therapeutic setting. A decrease of addictive symptoms and an improvement of executive control were observed in both cases. Discussion: Starting from these clinical observations, we provide a systematic review of the literature suggesting that BAs share similar neurobiological mechanisms to those underlying substance use disorders (SUD). Moreover, we discuss whether neurocircuit-based interventions, such as rTMS, might represent a potential effective treatment for BAs.
\end{abstract}

\section{KEYWORDS}

behavioral addiction (BA), internet gaming disorder (IGD), compulsive sexual behavior disorder (CSBD), repetitive transcranial magnetic stimulation (rTMS), dorsolateral prefrontal cortex (DLPFC)

\section{INTRODUCTION}

Behavioral addictions (BA) are increasingly recognized as a valid category of psychiatric disorder with relevant socio-cultural and economic implications (Robbins \& Clark, 2015). Despite the substantial progress in research on the neurobiology and clinical definition of addictive behaviors, our understanding of the potential neurobiological mechanisms related 
to specific non-substance addictive behaviors is still at an early stage and no proven specific pharmacotherapies are available (Marazziti, Presta, Baroni, Silvestri \& Dell'Osso, 2014). A large body of findings suggest that BAs share similar clinical, phenomenological, genetic, and neurobiological features with substance use disorders (SUD), supporting the inclusion of these disorders in the category of "Substance-related and Addictive disorders" of the Diagnostic and Statistical Manual of Mental Disorders, fifth edition (DSM-5) (Grant, Potenza, Weinstein, \& Gorelick, 2010). Similar to SUD, alterations in brain network activity related to reward processing, executive functions, inhibitory control response and habit formation and in neurotransmitter signaling, including dopamine, may underlie the development and maintenance of addictive behaviors (Brand et al., 2019). The identification of brain network activity dysfunctions could have important implications for the development of neurobiologically based treatments for both behavioral and drug addiction. Recently, repetitive transcranial magnetic stimulation (rTMS), is emerging as a promising therapeutic approach for SUD (Diana et al., 2017) and could potentially be an effective treatment for behavioral addictions. To our knowledge, we describe for the first time two cases of patients diagnosed with behavioral addictions (compulsive sexual behavior disorder for online porn use and internet gaming disorder) who received a multidisciplinary treatment including a rTMS protocol stimulating the left dorsolateral prefrontal cortex (1-DLPFC). Moreover, we provide a systematic review of the literature suggesting that BAs resemble SUD in many domains, including the phenomenology, the neurobiology and the clinical response and support the hypothesis that noninvasive neuromodulation techniques, such as rTMS might represent a potential effective treatment for BAs.

\section{CASE PRESENTATION}

\section{Patient 1}

The first patient, a 57-year-old sales representative Italian man, attended our outpatient clinic for addiction treatment from May 2019 to May 2020. His personal record stated an academic degree and a 22-years marriage with daughter.

At the first consultation, the patient complained of a loss of control over his online pornography use, started 15 years ago. After losing his job 5 years ago, he referred a very pervasive daily use which persisted until the month prior the visit: on average about 1 hour and a half a day with frequent binge-watching episodes, lasting 8 hours often without selfmaturbation. He was also absorbed by watching pornographic videos, spending a consistent amount of time to feel the desired level of excitement. This excessive level of engagement was reported as a way to relieve negative mood states as boredom, frustration, or depression. He progressively lost interest in other daily activities, including sexual activities, and repetitively lied to his wife and daughter to justify his behavior jeopardizing the relationship with them.
He also referred to have been abstinent from using pornography for the first time in 15 years during the last month. The period of abstinence was associated to intense feelings of sadness, anxiety, irritability. Furthermore, he was spending a growing amount of time in thinking of sex scenes, thus avoiding any stimuli which could trigger the urge for pornography, as watching romantic movies with his wife or using internet alone. When admitted to our clinic, the patient met full diagnostic criteria for Compulsive Sexual Behavior Disorder (CSBD) according to the 11th edition of the International Classification of Diseases (ICD-11) as assessed by a clinical psychiatrist specialized in addiction disorders (World Health Organization, 2018). A standardized clinical evaluation was conducted using the following self-reported scales: Beck Depression Inventory -II (BDI - II) (Beck, Steer, \& Brown, 1996), Pittsburgh Sleep Quality Index (PSQI) (Buysse et al., 1989), Self-rating Anxiety Scale (SAS) (Zung, 1971), Symptoms checklist 90 - Revised (SCL-90-R) (Derogatis, 1994) and a Visual Analogue Scale measuring craving for porn use (VAS craving). The patient was assessed at the first consultation, immediately before the rTMS treatment (nearly 20 days after the first consultation), after 5, 30, 60, 90 days of rTMS treatment and 1 month after the end of the treatment. The clinical outcomes scores of the patient during the observation period are summarized in Table 1 .

The patient reported symptoms of social anxiety disorder from a young age, such as hand sweating, palpitations, redness, stammering, hot flashes, and muscle tension in the presence of unfamiliar people or when he felt under judgment. Consistently, the standardized clinical assessment revealed mild to moderate anxiety severity levels (SAS Score $>45$, Table 1 ). His further medical history was not significant for any other mental disorder or medical condition. In the past, the patient referred unsuccessful psychotherapeutic attempts to face the addictive behavior and anxiety problems. To manage anxietyrelated symptoms, we started an off-label pharmacological therapy with propranolol (Inderal, AstraZeneca spa, Milan, Italy) $60 \mathrm{mg} / \mathrm{d}$. After 20 days, the patient reported a significant improvement of anxiety and depression symptoms with a reduction in palpitations and fainting feelings. He also noted to spend less time on thinking about sex scenes; however, the intrusive thoughts for pornography persisted in the everyday life situations (e.g. watching romantic movies with his wife) and the patient was continuously adopting avoidance behaviors. The persistence of cue-induced intrusive thoughts lead us to administer, following the acquisition of patient's informed consent approving the use of clinical data for research purposes, including the protocol approved by the Ethical Committee at Padua Teaching Hospital (protocol number: 4,743/ U6/19), an rTMS protocol treatment that has been associated to anti-craving effects in other addictive disorders (Gómez Pérez et al., 2020; Terraneo et al., 2016).

The rTMS protocol was carried out using a medical device (MagPro R30, MagVenture, Farum, Denmark) targeting the l-DLPFC (MNI coordinates: $\mathrm{x}=-50, \mathrm{y}=30, \mathrm{z}=$ 36) throughout an optical TMS Navigator (LOCALITE, St. Augustin, Germany) and a magnetic resonance image (MRI) template. Resting motor threshold (rMT) was determined 
Table 1. Patient 1 Clinical Outcome Scores

\begin{tabular}{|c|c|c|c|c|c|c|c|c|}
\hline & $\begin{array}{l}1^{\text {st }} \text { Consultation } \\
\text { Assessment }\end{array}$ & Baseline & Day 5 & $\begin{array}{c}\text { Day } \\
30\end{array}$ & $\begin{array}{c}\text { Day } \\
60\end{array}$ & $\begin{array}{c}\text { Day } \\
90\end{array}$ & $\begin{array}{l}1^{\text {st }} \text { month Follow- } \\
\text { up }\end{array}$ & Score Interpretation \\
\hline GSI & 75.00 & 50.62 & 43.98 & 40.67 & 40.33 & 40.33 & 41.66 & $\begin{array}{l}\text { 45-55: normal } \\
\text { 55-65: moderate } \\
\text { 65-75: severe }\end{array}$ \\
\hline PSQI & 4.00 & 3.00 & 5.00 & 1.00 & 3.00 & 5.00 & 4.00 & > 5: Poor Sleep Quality \\
\hline BDI-II & 19.00 & $-\S$ & 0.00 & 2.00 & 3.00 & 4.00 & 6.00 & $\begin{array}{l}\text { 14-18: mild-moderate } \\
\text { 19-29: moderate- } \\
\text { severe } \\
\text { 30-63: extremely } \\
\text { severe }\end{array}$ \\
\hline SAS & 50.00 & 41.25 & 35.00 & 36.25 & 36.25 & 35.00 & 40.00 & $\begin{array}{l}\text { 45-59: mild-moderate. } \\
\text { 60-55: moderate- } \\
\text { severe } \\
\geq 75 \text { : severe }\end{array}$ \\
\hline $\begin{array}{l}\text { VAS } \\
\text { craving }\end{array}$ & 9.00 & 1.00 & 0.00 & 0.00 & 0.00 & 0.00 & 0.00 & $0-9$ \\
\hline
\end{tabular}

Data are presented as raw scores, except for the GSI (T-Score) and the SAS (Index score). ${ }^{\S}$ BDI-II was not administered at day 5 because it refers to the last two weeks; BDI-II: Beck Depression Inventory-II; VAS craving: Visual Analogue Scale measuring craving for porn use induced by imagined environmental cues; GSI: Global Severity Index of the Symptoms checklist 90 - Revised; PSQI: Pittsburgh Sleep Quality Index; SAS: Self-rating Anxiety Scale.

using visual observation of muscle twitch (OM-MT) monthly. The stimulations parameters were set as previously reported (Terraneo et al., 2016): $15 \mathrm{~Hz}$ frequency, 100\% of rMT, 40 trains, 60 pulses per train, $15 \mathrm{~s}$ intertrain interval, and 2,400 pulses per session. The subject received 2 daily sessions for the first consecutive 5 days of treatment (10 sessions), and then 2 weekly sessions for the next 8 weeks. The time interval between two sessions each day was at least $30 \mathrm{~min}$. Moreover, individualized psychological counselling was daily provided by an addiction expert clinical psychologist. During the treatment no adverse event or subjective complaint was reported.

Following the rTMS treatment protocol, the patient has no longer manifested urges to use pornography, even in situations that were described as trigger (e.g. being alone in a hotel with his laptop or watching romantic movies with sex scenes). Pornography was no longer used to escape from unpleasant feelings and of intrusive thoughts about porn videos were not present. The patient also referred a consistent improvement of mood disturbances with normalization of anxiety levels and a significant enhancement of mood and a better relationship with his relatives.

At the 1-year follow-up examination, despite stressful situations, including the request for a salary increase, the unexpected diagnosis of Peyronie's disease and the Coronavirus Disease 2019 (COVID-19)-related lockdown, that could have represented a trigger for a relapse, the patient maintained the clinical improvement achieved. At this timepoint, it was not possible to perform the assessment using standardized self-reported scales due to the COVID-19 lockdown.

\section{Patient 2}

The second patient was a 21-year-old Italian male university student admitted to our addiction clinic from November 2018 to November 2019.
At the clinical intake interview, the patient referred an excessive online gaming activity, about 9 daily hours on weekdays and about 15 daily hours during the weekend, often losing the sense of time and neglecting other daily activities such as studying or engaging in social relationships. He was experiencing increasing difficulties in concentrating and being focused, as in 2-years of academic study he failed to pass his examinations. He was also less interested in hanging out and having physical interactions with friends and other people, rather preferring the social media chats to communicate with them. Over the time the patient attempted to suspend the online gaming activity experiencing anxiety, irritability and sadness associated to an increased need of gaming online and feeling guilty when not able to stop the use. The patient attributed the loss of control over gaming to an increased anxiety and sadness caused by a feeling of loneliness related to a recent move far from friends and family.

Investigating his medical history, an overuse of online gaming without negative consequences on his school performances or social interactions, was already present during the middle school, particularly when her mother was diagnosed with a severe neurological disease. Since childhood he referred mild problems to engage in stable peer relations and often feeling embarrassed in social contexts. The medical history was devoid of other significant mental or medical condition. A standardized clinical assessment was conducted at baseline and 3 and 12 months after the beginning of the rTMS treatment by using the following self-reported scales: BDI - II (Beck et al., 1996), PSQI (Buysse et al., 1989), SAS (Zung, 1971), SCL-90-R (Derogatis, 1994), Internet Gaming Disorder Scale - Short-Form (IGDS9-SF) (Monacis, Palo, Griffiths, M. D. \& Sinatra, 2016), Internet Addiction Test (IAT) (Servidio, 2017), Stroop Color Word Test-Short version (SCWT-SV) (Caffarra, Vezzadini, Dieci, Zonato, \& 
Venneri, 2002) and a Visual Analogue Scale measuring current craving for gaming (VAS craving). The clinical outcomes scores of the patient during treatment are shown in Table 2. At baseline, a high degree of internet gaming disorder was present as indicated by the high IGDS9-SF score (> 21, Table 2).

We hypothesized that the persistent and dysfunctional addictive-like behavior could benefit from a rTMS protocol treatment over the l-DLPFC. After obtaining the informed patient's consent approving the use of clinical data for research purposes, including the protocol approved by the Ethical Committee at Padua Teaching Hospital (protocol number: 4,743/U6/19), an rTMS protocol treatment, as previously described was administered. He also received psychological support by a clinical psychologist trained in addiction, whereas no pharmacological treatment was prescribed. No subjective complaint or adverse event was observed during or after rTMS protocol. After the first week of treatment, the patient reported a significant reduction of the time spent on internet, up to only 2 hours per week. Internet was no longer used for gaming, rather for study purposes. Furthermore, the patient was experiencing an unprecedent capacity to interrupt internet use once started and the resumption to be engaged in healthy leisure activities outside (e.g. going to the bookstore, hanging out with friends). At the end of the 9-weeks rTMS treatment, the subjective clinical improvement persisted and no irritability, anxiety, or recurrent thoughts about online gaming were reported. He managed to reorganize his daily routine activity mainly focusing on his academic goals, re-establishing old friendship or meeting new people. However, the progressive clinical improvement of gaming maladaptive behavior was accompanied by mood deflection and psychological distress related to a pre-existing and marked perfectionism attitude and low self-esteem, manifesting especially during social interaction. Therefore, the patient was referred to a psychotherapist. At the 1 year follow up, he demonstrated a significant improvement of his emotional state and relational skills, while symptoms of internet gaming addiction were no longer present.

\section{DISCUSSION}

Here, we describe two cases of BA, online pornography addiction and internet gaming disorder, diagnosed by applying the existing criteria for "substance-related and addictive disorders" in the Diagnostic and Statistical Manual of Mental Disorders Fifth Edition (Vinet \& Zhedanov, 2010) and the CSBD criteria in the International Classification of Diseases Eleventh Edition (World Health Organization, 2018). Both patients came to our attention complaining of symptoms resembling those of addictive disorders, including a pattern of persistent and excessive behavior (porn watching or internet gaming), several failed attempt to control the behavior, tolerance, and craving. This clinical picture was accompanied of withdrawal symptoms, including negative mood and increased stress reactivity, and an impairment of

Table 2. Patient 2 Clinical Outcome Scores

\begin{tabular}{|c|c|c|c|c|}
\hline & Baseline & Day 90 & 1-year Follow-up & Score Interpretation \\
\hline GSI & 51.57 & 61.14 & 48.04 & $\begin{array}{l}\text { 45-55: normal } \\
\text { 55-65: moderate } \\
\text { 65-75: severe }\end{array}$ \\
\hline PSQI & 4.00 & 6.00 & 1.00 & > 5: Poor Sleep Quality \\
\hline BDI-II & 13.00 & 25.00 & 4.00 & $\begin{array}{l}\text { 14-18: mild-moderate } \\
\text { 19-29: moderate-severe } \\
\text { 30-63: extremely severe }\end{array}$ \\
\hline SAS & 32.50 & 50.00 & 36.25 & $\begin{array}{l}\text { 45-59: mild-moderate } \\
\text { 60-55: moderate-severe } \\
\geq 75: \text { severe }\end{array}$ \\
\hline VAS craving & 75.00 & 55.00 & 5.00 & $0-100$ \\
\hline IGDS9-SF & 32.00 & $16.00^{*}$ & 0.00 & $>21$ : Disordered gaming \\
\hline IAT & 59.00 & 30.00 & 26.00 & $\begin{array}{l}\text { 31-49: mild problems } \\
\text { 50-79: moderate problems } \\
\text { 80-100: severe problems }\end{array}$ \\
\hline $\begin{array}{l}\text { SCWT-SV: } \\
\text { Interference time score }\end{array}$ & 4.00 & 4.00 & 4.00 & $\begin{array}{l}\text { 0: below the norm } \\
\text { 1: lower limit of the norm } \\
2-4 \text { : within the norm }\end{array}$ \\
\hline $\begin{array}{l}\text { SCWT-SV: } \\
\text { Interference error score }\end{array}$ & 1.00 & 1.00 & 4.00 & $\begin{array}{l}\text { 0: below the norm } \\
\text { 1: lower limit of the norm } \\
2-4 \text { : within the norm }\end{array}$ \\
\hline
\end{tabular}

Data are presented as raw scores, except for the GSI (T-Score), the SAS (Index score) and the SCWT-SV (Equivalent score). ${ }^{\star}$ This scale was administered at day 90 referring to the last three months for clinical reasons; BDI-II: Beck Depression Inventory-II; VAS craving: Visual Analogue Scale measuring current craving for gaming; GSI: Global Severity Index of the Symptoms checklist 90 - Revised; PSQI: Pittsburgh Sleep Quality Index; SAS: Self-rating Anxiety Scale; IGDS9-SF: Internet Gaming Disorder Scale - Short-Form; IAT: Internet Addiction Test; SCWT-SV: Stroop Color Word Test-Short version. 
their personal, professional, and social life. In contrast to SUD, no approved medication for the treatment of behavioral addictions is available (Mouaffak et al., 2017). However, since underlying neural processes as well as clinical symptoms are found to be similar in BA and SUD (Grant et al., 2010), we sought that non-invasive neuromodulation intervention might be a promising candidate for the treatment of behavioral addictions as well. To our knowledge, these two cases are the first description of internet addiction treated with a rTMS protocol treatment targeting the 1DLPFC.

There is a general consensus suggesting etiological, cognitive and personality features similarities between SUD and non-substance related addictions (Brand et al., 2019; Brand, Young, Laier, Wölfling, \& Potenza, 2016). However, the pattern of impulsivity and compulsivity of addictive behaviors might underlie different endophenotype that can critically differentiate SUD from non-substance related addiction (Wareham \& Potenza, 2010). Although additional studies employing larger samples and a wider array of imaging modalities are needed to investigate the neurobiological similarities and differences of individuals with SUD and behavioral addiction, these internet-use related disorders share with substance addiction personality traits, including impulsivity, sensation seeking, and neuroticism (Antons \& Brand, 2018; Dayan, Bernard, Olliac, Mailhes, \& Kermarrec, 2010; Ko et al., 2014; Lejuez et al., 2010; Liu et al., 2014; Ma et al., 2010; Mehroof \& Griffiths, 2010; Müller, Beutel, Egloff \& Wölfling, 2014; Uhl et al., 2008) and escapism to avoid hassles and distress as negative reinforcement mechanism for maintaining abuse behaviour (Baker, Piper, McCarthy, Majeskie, \& Fiore, 2004; Hagström \& Kaldo, 2014). Interestingly, both patients reported escaping from unpleasant feelings as an essential driving motivation to engage in overuse behaviour.

Similarly, cognitive disturbances, typically associated to SUD, such as impaired decision-making ability, biases toward addiction related stimuli and lower response inhibition (Bechara \& Martin, 2004; Field, Mogg \& Bradley, 2004, Field, Munafò \& Franken, 2009; Goldstein \& Volkow, 2011; Jovanovski, Erb \& Zakzanis, 2005; Koob \& Volkow, 2016; Spronk, van Wel, Ramaekers \& Verkes, 2013; VerdejoGarcía, Bechara, Recknor \& Perez-Garcia, 2006) have also been reported in people with non-substance addictions (Mechelmans et al., 2014; Pawlikowski \& Brand, 2011; Zhou, Yuan \& Yao, 2012). The cognitive function impairment might be related to an imbalance between the increasing incentive-oriented urges and the decreasing situation-specific inhibitory control over these urges, that plays a key role for the onset and maintenance of addictive behaviors (Brand et al., 2019). Furthermore, brain imaging studies have demonstrated similar alterations in cortico-limbic-striatal and prefrontal control circuits, involving ventral striatum, amygdala, and dorsolateral prefrontal areas, in either SUD or BA indicating an enhanced activation of these networks during cue-induced craving processing but a blunted response during executive control processing (Brand, Snagowski, Laier, \& Maderwald, 2016; Chen et al., 2015;
Han, Hwang \& Renshaw, 2010; Ko et al., 2014, 2009; Liu et al., 2014; Zilverstand, Huang, Alia-Klein \& Goldstein, 2018).

Thus, prolonged exposure to addictive agents or engaging in persistent addictive behaviors are associated to neural circuit dysfunctions implicated in reward, salience attribution, motivation, inhibitory control, learning and memory consolidation, suggesting a common neurobiological basis for SUD and BA. Preclinical studies manipulating corticostriatal circuits by using optogenetics provided foundation to explore neuromodulation as an effective treatment for stimulant use disorder (Chen et al., 2013). rTMS represents a novel and promising therapeutic approach for addiction (Spagnolo, Gómez Pérez, Terraneo, Gallimberti \& Bonci, 2019) as it modulates neural activity in the short and long term period by inducing neuroplastic changes (Fox, Halko, Eldaief \& Pascual-Leone, 2012). This approach has been associated to clinically relevant behavioral changes in patients with addictive disorders (Ekhtiari et al., 2019), affecting craving, intake and relapse (Diana et al., 2017). To date, high-frequency rTMS protocols over the dorsolateral prefrontal cortex, a key node for the executive control network (Shirer, Ryali, Rykhlevskaia, Menon \& Greicius, 2012), are effective in reducing craving, substance consumption and withdrawal symptoms of SUD, including alcohol (Addolorato et al., 2017; Mishra, Nizamie, Das \& Praharaj, 2010; Mishra, Praharaj, Katshu, Sarkar \& Nizamie, 2015), tobacco (Amiaz, Levy, Vainiger, Grunhaus \& Zangen, 2009; Eichhammer et al., 2003; Hayashi, Ko, Strafella \& Dagher, 2013; Johann et al., 2003; Li et al., 2013), cocaine (Gómez Pérez et al., 2020; Hanlon et al., 2015; Madeo et al., 2020; Pettorruso et al., 2019; Politi, Fauci, Santoro, \& Smeraldi, 2008; Sanna et al., 2019; Steele, Maxwell, Ross, Stein, \& Salmeron, 2019; Terraneo et al., 2016), methamphetamine (Liang, Wang \& Yuan, 2018; Su et al., 2017) and heroin (Shen et al., 2016). Preliminary findings also suggest that these protocols might ameliorate gambling disorder symptoms (Cardullo et al., 2019; Pettorruso et al., 2020).

In our cases, both patients regained the ability to control their behavioural urges and craving-like thoughts after the rTMS protocol treatment. Following the treatment, they also reported an internet use for professional purposes, as university or work assignments. We hypothesized that the clinical improvement of behavioral addictive symptoms might be related to the rTMS-induced neuromodulatory effect over the brain networks areas mediating response inhibition and control of impulsive behaviour (Dunlop, Hanlon \& Downar, 2017; Gorelick, Zangen, \& George, 2014).

In medicine and in some therapeutic areas, including psychiatric disorders, a possible placebo response might influence the final outcome of clinical trials. From a psychological point of view the placebo response may be triggered by various interrelated environmental and psychosocial factors, such as patient's expectations of the benefit of a treatment, behavioural conditioning, and the quality of the patient-physician relationship (Enck, Bingel, Schedlowski \& Rief, 2013). We took into account all these elements and we adopted some procedures to minimize as much as possible 
the placebo effect. Both patients were given clear and exhaustive information regarding the off-label use of the rTMS treatment, for whom no evidence of efficacy is still available in the literature. This approach allowed to balance the patients' expectations towards the treatment final outcome. Indeed, Patient 1 started an off-label pharmacological treatment with the beta-blocker propranolol before the rTMS protocol obtaining an improvement only of his anxiety-related symptoms whereas the cue-induced intrusive thoughts persisted.

Notably, the clinical recovery persisted at 1-year followup after the treatment although both patients experienced unpleasant situations, recognized as triggers for inducing craving for gaming or porn watching in the past. This longlasting clinical improvement over the addictive behaviours with no relapses in both patients is in support of a minimized placebo response and is in line with our previous findings showing that rTMS treatment is accompanied by long-lasting reductions of substance consumption behaviours in a large cohort of patients with cocaine use disorder clinically followed-up for 2 years and 8 months (Madeo et al., 2020).

Patient 2 required a psychotherapic support to address some of his personality features, including perfectionism and low-self-esteem, that emerged during the treatment. Several finding now suggest that cognitive, personality and mood features are associated with internet addiction (Şenormanc1 et al., 2014; Younes et al., 2016) and might play a role in developing addictive behavior as a coping strategy to be relieved from their negative beliefs (Şenormanc1 et al., 2014). The improvement of cortical function through the neuromodulatory intervention might have allowed a more successful psychotherapeutic approach (Bajbouj \& Padberg, 2014) (Donse, Padberg, Sack, Rush \& Arns, 2018), as seen in patient 2.

Likewise, in patient 2 we observed prominent autonomic symptoms associated with social anxiety, a frequent comorbidity in internet-related disorders (Bernardi \& Pallanti, 2009). We efficaciously controlled the autonomic-related symptomatology by administering propranolol, a nonselective beta-blocker blocking the catecholamines action through the beta- 1 and beta-2 adrenergic receptors (Routledge \& Shand, 1979). Propranolol effectively suppresses the autonomic hyperactivity and hyperarousal associated with anxiety disorder, reducing thereby its physical symptoms (Brantigan, Brantigan, \& Joseph, 1982; Mealy et al., 1996). Consistently with the pharmacological effects, after 20 days of treatment patient 1 experienced an improvement of autonomic-related symptoms but the intrusive thinking and cue-induced craving persisted. A substantial improvement of addictive related behaviors was observed after the rTMS treatment. In both substance and porn addictions, increased reactivity towards appetitive cues is associated with a reduced connectivity between dorsal prefrontal regions, mediating cognitive control, and limbic areas, relevant for the motivational salience (Berlingeri et al., 2017; Klucken, Wehrum-Osinsky, Schweckendiek, Kruse \& Stark, R., 2016; Ma et al., 2010; Schmidt et al., 2017). Thus, the rTMS treatment strategy focused on amplifying activity in frontalstriatal circuits might improve cognitive functions and reduce craving (Hanlon et al., 2015).

In conclusion, the reported cases describe how BAs, sharing clinical core symptoms with SUD may benefit from a neuromodulatory intervention using a rTMS protocol stimulating the DLPFC. Hence, further studies are needed to validate these preliminary observations and investigate whether rTMS could be a treatment option for non-substance addictions, such as internet gaming disorders or online porn-addiction.

Funding sources: Nothing declared.

Authors' contribution: DC and SS followed the patients up during all the study period and collected the clinical data. GM, DC, SC, and LGP supervised and interpreted the data. GM, DC and LGP co-wrote the manuscript. LG, NC, MS, SCh critically reviewed the manuscript. All authors contributed to and have approved the final manuscript.

Conflict of interest: The authors declare no conflict of interest.

Acknowledgements: The authors would also like to thank all personnel of the addiction specialty outpatient clinic from Padua and Milan (Italy) for their invaluable collaboration in the collection of data.

\section{REFERENCES}

Addolorato, G., Antonelli, M., Cocciolillo, F., Vassallo, G. A., Tarli, C., Sestito, L., et al. (2017). Deep transcranial magnetic stimulation of the dorsolateral prefrontal cortex in alcohol use disorder patients: Effects on dopamine transporter availability and alcohol intake. European Neuropsychopharmacology: The Journal of the European College of Neuropsychopharmacology, 27(5), 450-461. https://doi.org/10.1016/j.euroneuro.2017.03.008.

Amiaz, R., Levy, D., Vainiger, D., Grunhaus, L., \& Zangen, A. (2009). Repeated high-frequency transcranial magnetic stimulation over the dorsolateral prefrontal cortex reduces cigarette craving and consumption. Addiction, 104, 653-660. https://doi. org/10.1111/j.1360-0443.2008.02448.x.

Antons, S., \& Brand, M. (2018). Trait and state impulsivity in males with tendency towards Internet-pornography-use disorder. Addictive Behaviors, 79, 171-177. https://doi.org/10.1016/j. addbeh.2017.12.029.

Bajbouj, M., \& Padberg, F. (2014). A perfect match: Noninvasive brain stimulation and psychotherapy. European Archives of Psychiatry and Clinical Neuroscience, 264(1), 27-33. https://doi. org/10.1007/s00406-014-0540-6.

Baker, T. B., Piper, M. E., McCarthy, D. E., Majeskie, M. R., \& Fiore, M. C. (2004). Addiction motivation reformulated: An affective processing model of negative reinforcement. Psychological Review, 111(1), 33. https://doi.org/10.1037/0033-295X. 111.1.33. 
Bechara, A., \& Martin, E. M. (2004). Impaired decision making related to working memory deficits in individuals with substance addictions. Neuropsychology, 18(1), 152. https://doi.org/ 10.1037/0894-4105.18.1.152.

Beck, A., Steer, R., \& Brown, G. (1996). Beck depression inventoryII. San Antonio, TX: Psychological Corporation. http://www. pearsonclinical.com/psychology/products/100000159/beckdepression-inventoryii-bdi-ii.html?Pid=015-8018-370.

Berlingeri, M., Losasso, D., Girolo, A., Cozzolino, E., Masullo, T., Scotto, M., et al. (2017). Resting state brain connectivity patterns before eventual relapse into cocaine abuse. Behavioural Brain Research, 327, 121-132. https://doi.org/10.1016/j.bbr.2017.01.002.

Bernardi, S., \& Pallanti, S. (2009). Internet addiction: A descriptive clinical study focusing on comorbidities and dissociative symptoms. Comprehensive Psychiatry, 50(6), 510-516. https:// doi.org/10.1016/j.comppsych.2008.11.011.

Brand, M., Snagowski, J., Laier, C., \& Maderwald, S. (2016). Ventral striatum activity when watching preferred pornographic pictures is correlated with symptoms of Internet pornography addiction. NeuroImage, 129, 224-232. https://doi.org/10.1016/j. neuroimage.2016.01.033.

Brand, M., Wegmann, E., Stark, R., Müller, A., Wölfling, K., Robbins, T. W., et al. (2019). The Interaction of Person-AffectCognition-Execution (I-PACE) model for addictive behaviors: Update, generalization to addictive behaviors beyond Internetuse disorders, and specification of the process character of addictive behaviors. Neuroscience \& Biobehavioral Reviews, 104, 1-10. https://doi.org/10.1016/j.neubiorev.2019.06.032.

Brand, M., Young, K. S., Laier, C., Wölfling, K., \& Potenza, M. N. (2016). Integrating psychological and neurobiological considerations regarding the development and maintenance of specific Internet-use disorders: An Interaction of Person-AffectCognition-Execution (I-PACE) model. Neuroscience \& Biobehavioral Reviews, 71, 252-266. https://doi.org/10.1016/j. neubiorev.2016.08.033.

Brantigan, C. O., Brantigan, T. A., \& Joseph, N. (1982). Effect of beta blockade and beta stimulation on stage fright. The American Journal of Medicine, 72(1), 88-94. https://doi.org/10.1016/ 0002-9343(82)90592-7.

Buysse, D. J., Reynolds, C. F., Monk, T. H., Berman, S. R., Kupfer, D. J., III, C. F. R., et al. (1989). The Pittsburgh Sleep quality Index: A new instrument for psychiatric practice and research. Psychiatry Research, 28(2), 193-213. https://doi.org/10.1016/ 0165-1781(89)90047-4.

Caffarra, P., Vezzadini, G., Dieci, F., Zonato, F., \& Venneri, A. (2002). Una versione abbreviata del test di Stroop: Dati normativi nella popolazione italiana. Nuova Rivista Di Neurologia, 12(4), 111-115.

Cardullo, S., Gómez Pérez, L. J., Marconi, L., Terraneo, A., Gallimberti, L., Bonci, A., et al. (2019). Clinical improvements in comorbid gambling/cocaine use disorder (GD/CUD) patients undergoing repetitive transcranial magnetic stimulation (rTMS). Journal of Clinical Medicine, 8(6), 768. https://doi.org/ $10.3390 / \mathrm{jcm} 8060768$

Chen, C., Huang, M., Yen, J., Chen, C., Liu, G., Yen, C., et al. (2015). Brain correlates of response inhibition in Internet gaming disorder. Psychiatry and Clinical Neurosciences, 69(4), 201-209. https://doi.org/10.1111/pen.12224.
Chen, B. T., Yau, H. J., Hatch, C., Kusumoto-Yoshida, I., Cho, S. L., Hopf, F. W., et al. (2013). Rescuing cocaine-induced prefrontal cortex hypoactivity prevents compulsive cocaine seeking. $\mathrm{Na}$ ture, 496, 359-362. https://doi.org/10.1038/nature12024.

Dayan, J., Bernard, A., Olliac, B., Mailhes, A.-S., \& Kermarrec, S. (2010). Adolescent brain development, risk-taking and vulnerability to addiction. Journal of Physiology Paris, 104(5), 279-286. https://doi.org/10.1016/j.jphysparis.2010.08.007.

Derogatis, L. R. (1994). Symptom Checklist-90-R (SCL-90-R): Administration, scoring, and procedures manual. 3rd ed. Minneapolis, MN: NCS Pearson.

Diana, M., Raij, T., Melis, M., Nummenmaa, A., Leggio, L., \& Bonci, A. (2017). Rehabilitating the addicted brain with transcranial magnetic stimulation. Nature Reviews Neuroscience, 18(11), 685-693. https://doi.org/10.1038/nrn.2017.113.

Donse, L., Padberg, F., Sack, A. T., Rush, A. J., \& Arns, M. (2018). Simultaneous rTMS and psychotherapy in major depressive disorder: Clinical outcomes and predictors from a large naturalistic study. Brain Stimulation, 11(2), 337-345. https://doi. org/10.1016/j.brs.2017.11.004.

Dunlop, K., Hanlon, C. A., \& Downar, J. (2017). Noninvasive brain stimulation treatments for addiction and major depression. Annals of the New York Academy of Sciences, 1394(1), 31-54. https://doi.org/10.1111/nyas.12985.

Eichhammer, P., Johann, M., Kharraz, A., Binder, H., Pittrow, D., Wodarz, N., et al. (2003). High-frequency repetitive transcranial magnetic stimulation decreases cigarette smoking. Journal of Clinical Psychiatry, 64(8), 951-953.

Ekhtiari, H., Tavakoli, H., Addolorato, G., Baeken, C., Bonci, A., Campanella, S., et al. (2019). Transcranial electrical and magnetic stimulation (tES and TMS) for addiction medicine: A consensus paper on the present state of the science and the road ahead. Neuroscience \& Biobehavioral Reviews, 104, 118-140.

Enck, P., Bingel, U., Schedlowski, M., \& Rief, W. (2013). The placebo response in medicine: Minimize, maximize or personalize? Nature Reviews Drug Discovery, 12(3), 191-204.

Field, M., Mogg, K., \& Bradley, B. P. (2004). Cognitive bias and drug craving in recreational cannabis users. Drug and Alcohol Dependence, 74(1), 105-111. https://doi.org/10.1016/j. drugalcdep.2003.12.005.

Field, M., Munafò, M. R., \& Franken, I. H. A. (2009). A meta-analytic investigation of the relationship between attentional bias and subjective craving in substance abuse. Psychological Bulletin, 135(4), 589-607. https://doi.org/10.1037/a0015843.

Fox, M. D., Halko, M. A., Eldaief, M. C., \& Pascual-Leone, A. (2012). Measuring and manipulating brain connectivity with resting state functional connectivity magnetic resonance imaging (fcMRI) and transcranial magnetic stimulation (TMS). NeuroImage, 62(4), 2232-2243. https://doi.org/10.1016/j. neuroimage.2012.03.035.

Goldstein, R. Z., \& Volkow, N. D. (2011). Dysfunction of the prefrontal cortex in addiction: Neuroimaging findings and clinical implications. Nature Reviews Neuroscience. https://doi. org/10.1038/nrn3119.

Gómez Pérez, L. J., Cardullo, S., Cellini, N., Sarlo, M., Monteanni, T., Bonci, A., et al. (2020). Sleep quality improves during treatment with repetitive transcranial magnetic stimulation (rTMS) in patients with cocaine use disorder: A retrospective 
observational study. BMC Psychiatry, 20(153), 1-12. https://doi. org/10.1186/s12888-020-02568-2.

Gorelick, D. A., Zangen, A., \& George, M. S. (2014). Transcranial magnetic stimulation in the treatment of substance addiction. Annals of the New York Academy of Sciences, 1327(1), 79-93. https://doi.org/10.1111/nyas.12479.

Grant, J. E., Potenza, M. N., Weinstein, A., \& Gorelick, D. A. (2010). Introduction to behavioral addictions. The American Journal of Drug and Alcohol Abuse, 36(5), 233-241. https://doi. org/10.3109/00952990.2010.491884.

Hagström, D., \& Kaldo, V. (2014). Escapism among players of MMORPGs-conceptual clarification, its relation to mental health factors, and development of a new measure. Cyberpsychology, Behavior and Social Networking, 17(1), 19-25. https://doi.org/10.1089/cyber.2012.0222.

Han, D. H., Hwang, J. W., \& Renshaw, P. F. (2010). Bupropion sustained release treatment decreases craving for video games and cue-induced brain activity in patients with internet video game addiction. Experimental and Clinical Psychopharmacology. https://doi.org/10.1037/a0020023.

Hanlon, C. A., Dowdle, L. T., Austelle, C. W., Devries, W., Mithoefer, O., Badran, B. W., et al. (2015). What goes up, can come down: Novel brain stimulation paradigms may attenuate craving and craving-related neural circuitry in substance dependent individuals. Brain Research, 1628, 199-209. https:// doi.org/10.1016/j.brainres.2015.02.053.

Hayashi, T., Ko, J. H., Strafella, A. P., \& Dagher, A. (2013). Dorsolateral prefrontal and orbitofrontal cortex interactions during self-control of cigarette craving. Proceedings of the $\mathrm{Na}$ tional Academy of Sciences, 110(11), 4422-4427. https://doi.org/ 10.1073/pnas.1212185110.

Johann, M., Wiegand, R., Kharraz, A., Bobbe, G., Sommer, G., Hajak, G., et al. (2003). Transcranial magnetic stimulation for nicotine dependence. Psychiatrische Praxis, 30(Suppl 2), S129S131. http://www.ncbi.nlm.nih.gov/pubmed/14509058.

Jovanovski, D., Erb, S., \& Zakzanis, K. K. (2005). Neurocognitive deficits in cocaine users: A quantitative review of the evidence. Journal of Clinical and Experimental Neuropsychology, 27(2), 189-204. https://doi.org/10.1080/ 13803390490515694.

Klucken, T., Wehrum-Osinsky, S., Schweckendiek, J., Kruse, O., \& Stark, R. (2016). Altered appetitive conditioning and neural connectivity in subjects with compulsive sexual behavior. The Journal of Sexual Medicine, 13(4), 627-636. https://doi.org/10. 1016/j.jsxm.2016.01.013.

Ko, C.-H., Hsieh, T.-J., Chen, C.-Y., Yen, C.-F., Chen, C.-S., Yen, J.Y., et al. (2014). Altered brain activation during response inhibition and error processing in subjects with internet gaming disorder: A functional magnetic imaging study. European Archives of Psychiatry and Clinical Neuroscience, 264(8), 661-672. https://doi.org/10.1007/s00406-013-0483-3.

Ko, C.-H., Liu, G.-C., Hsiao, S., Yen, J.-Y., Yang, M.-J., Lin, W.-C., et al. (2009). Brain activities associated with gaming urge of online gaming addiction. Journal of Psychiatric Research, 43(7), 739-747. https://doi.org/10.1016/j.jpsychires.2008.09.012.

Koob, G. F., \& Volkow, N. D. (2016). Neurobiology of addiction: A neurocircuitry analysis. The Lancet Psychiatry, 3(8), 760-773. https://doi.org/10.1016/S2215-0366(16)00104-8.
Lejuez, C. W., Magidson, J. F., Mitchell, S. H., Sinha, R., Stevens, M. C., \& De Wit, H. (2010). Behavioral and biological indicators of impulsivity in the development of alcohol use, problems, and disorders. Alcoholism: Clinical and Experimental Research, 34(8), 1334-1345. https://doi.org/10.1111/j.1530-0277.2010. 01217.x.

Liang, Y., Wang, L., \& Yuan, T. F. (2018). Targeting withdrawal symptoms in men addicted to methamphetamine with transcranial magnetic stimulation: A randomized clinical trial. JAMA Psychiatry, 75(11), 1199-1201. https://doi.org/10.1001/ jamapsychiatry.2018.2383.

Li, X., Hartwell, K. J., Owens, M., Lematty, T., Borckardt, J. J., Hanlon, C. A., et al. (2013). Repetitive transcranial magnetic stimulation of the dorsolateral prefrontal cortex reduces nicotine cue craving. Biological Psychiatry, 73(8), 714-720. https:// doi.org/10.1016/j.biopsych.2013.01.003.

Liu, G.-C., Yen, J.-Y., Chen, C.-Y., Yen, C.-F., Chen, C.-S., Lin, W.C., et al. (2014). Brain activation for response inhibition under gaming cue distraction in internet gaming disorder. The Kaohsiung Journal of Medical Sciences, 30(1), 43-51. https://doi. org/10.1016/j.kjms.2013.08.005.

Madeo, G., Terraneo, A., Cardullo, S., Gómez Pérez, L. J., Cellini, N., Sarlo, M., et al. (2020). Long-term outcome of repetitive transcranial magnetic stimulation in a large cohort of patients with cocaine-use disorder: An observational study. Frontiers in Psychiatry, 11(158). https://doi.org/10.3389/fpsyt.2020.00158.

Ma, N., Liu, Y., Li, N., Wang, C. X., Zhang, H., Jiang, X. F., et al. (2010). Addiction related alteration in resting-state brain connectivity. NeuroImage, 49(1), 738-744. https://doi.org/10.1016/ j.neuroimage.2009.08.037.

Marazziti, D., Presta, S., Baroni, S., Silvestri, S., \& Dell'Osso, L. (2014). Behavioral addictions: A novel challenge for psychopharmacology. CNS Spectrums, 19(6), 486-495. https://doi.org/ 10.1017/S1092852913001041.

Mealy, K., Ngeh, N., Gillen, P., Fitzpatrick, G., Keane, F. B., \& Tanner, A. (1996). Propranolol reduces the anxiety associated with day case surgery. The European Journal of Surgery Acta Chirurgica, 162(1), 11-14.

Mechelmans, D. J., Irvine, M., Banca, P., Porter, L., Mitchell, S., Mole, T. B., et al. (2014). Enhanced attentional bias towards sexually explicit cues in individuals with and without compulsive sexual behaviours. PloS One, 9(8). https://doi.org/10.1371/ journal.pone.0105476.

Mehroof, M., \& Griffiths, M. D. (2010). Online gaming addiction: The role of sensation seeking, self-control, neuroticism, aggression, state anxiety, and trait anxiety. Cyberpsychology, Behavior and Social Networking, 13(3), 313-316. https://doi. org/10.1089/cyber.2009.0229.

Mishra, B.R., Nizamie, S. H., Das, B., \& Praharaj, S. K. (2010). Efficacy of repetitive transcranial magnetic stimulation in alcohol dependence: A sham-controlled study. Addiction, 105, 49-55. https://doi.org/10.1111/j.1360-0443.2009.02777.x.

Mishra, B.R., Praharaj, S. K., Katshu, M. Z. U. H., Sarkar, S., \& Nizamie, S. H. (2015). Comparison of anticraving efficacy of right and left repetitive transcranial magnetic stimulation in alcohol dependence: A randomized double-blind study. The Journal of Neuropsychiatry and Clinical Neurosciences, 27(1), e54-e59. https://doi.org/10.1176/appi.neuropsych.13010013. 
Monacis, L., Palo, V. de, Griffiths, M. D., \& Sinatra, M. (2016). Validation of the internet gaming disorder scale - short-form (IGDS9-SF) in an Italian-speaking sample. Journal of Behavioral Addictions. https://doi.org/10.1556/2006.5.2016.083.

Mouaffak, F., Leite, C., Hamzaoui, S., Benyamina, A., Laqueille, X., \& Kebir, O. (2017). Naltrexone in the treatment of broadly defined behavioral addictions: A review and meta-analysis of randomized controlled trials. European Addiction Research, 23(4), 204-210. https://doi.org/10.1159/000480539.

Müller, K. W., Beutel, M. E., Egloff, B., \& Wölfling, K. (2014). Investigating risk factors for internet gaming disorder: A comparison of patients with addictive gaming, pathological gamblers and healthy controls regarding the big five personality traits. European Addiction Research, 20(3), 129-136. https://doi. org/10.1159/000355832.

Pawlikowski, M., \& Brand, M. (2011). Excessive internet gaming and decision making: Do excessive world of warcraft players have problems in decision making under risky conditions? Psychiatry Research, 188(3), 428-433. https://doi.org/10.1016/j. psychres.2011.05.017.

Pettorruso, M., Martinotti, G., Montemitro, C., De Risio, L., Spagnolo, P. A., Gallimberti, L., et al. (2020). Multiple sessions of high-frequency repetitive transcranial magnetic stimulation as a potential treatment for gambling addiction: A 3-month, feasibility study. European Addiction Research, 26(1), 52-56. https://doi.org/10.1159/000504169.

Pettorruso, M., Martinotti, G., Santacroce, R., Montemitro, C., Fanella, F., \& Di Giannantonio, M. (2019). rTMS reduces psychopathological burden and cocaine consumption in treatment-seeking subjects with Cocaine Use Disorder: An open label, feasibility study. Frontiers in Psychiatry, 10, 621. https:// doi.org/10.3389/fpsyt.2019.00621.

Politi, E., Fauci, E., Santoro, A., \& Smeraldi, E. (2008). Daily sessions of transcranial magnetic stimulation to the left prefrontal cortex gradually reduce cocaine craving. The American Journal on Addictions, 17(4), 345-346. https://doi.org/10.1080/ 10550490802139283.

Robbins, T. W., \& Clark, L. (2015). Behavioral addictions. Current Opinion in Neurobiology, 30, 66-72. https://doi.org/10.1016/j. conb.2014.09.005.

Routledge, P. A., \& Shand, D. G. (1979). Clinical pharmacokinetics of propranolol. Clinical Pharmacokinetics, 4(2), 73-90. https:// doi.org/10.2165/00003088-197904020-00001.

Sanna, A., Fattore, L., Badas, P., Corona, G., Cocco, V., \& Diana, M. (2019). Intermittent theta burst stimulation of the prefrontal cortex in cocaine use disorder: A pilot study. Frontiers in Neuroscience, 13, 765.

Schmidt, C., Morris, L. S., Kvamme, T. L., Hall, P., Birchard, T., \& Voon, V. (2017). Compulsive sexual behavior: Prefrontal and limbic volume and interactions. Human Brain Mapping, 38(3), 1182-1190. https://doi.org/10.1002/hbm.23447.

Şenormancı, Ö., Saraçlı, Ö., Atasoy, N., Şenormancı, G., Koktürk, F., \& Atik, L. (2014). Relationship of Internet addiction with cognitive style, personality, and depression in university students. Comprehensive Psychiatry, 55(6), 1385-1390. https://doi. org/10.1016/j.comppsych.2014.04.025.

Servidio, R. (2017). Assessing the psychometric properties of the internet addiction test: A study on a sample of Italian university students. Computers in Human Behavior, 68, 17-29. https://doi. org/10.1016/j.chb.2016.11.019.

Shen, Y., Cao, X., Tan, T., Shan, C., Wang, Y., Pan, J., et al. (2016). $10-\mathrm{Hz}$ repetitive transcranial magnetic stimulation of the left dorsolateral prefrontal cortex reduces heroin cue craving in long-term addicts. Biological Psychiatry, 80(3), e13-e14. https:// doi.org/10.1016/j.biopsych.2016.02.006.

Shirer, W. R., Ryali, S., Rykhlevskaia, E., Menon, V., \& Greicius, M. D. (2012). Decoding subject-driven cognitive states with wholebrain connectivity patterns. Cerebral Cortex, 22(1), 158-165.

Spagnolo, P. A., Gómez Pérez, L. J., Terraneo, A., Gallimberti, L., \& Bonci, A. (2019). Neural correlates of cue-and stress-induced craving in gambling disorders: Implications for transcranial magnetic stimulation interventions. European Journal of Neuroscience, 1-14.

Spronk, D. B., van Wel, J. H. P., Ramaekers, J. G., \& Verkes, R. J. (2013). Characterizing the cognitive effects of cocaine: A comprehensive review. Neuroscience and Biobehavioral Reviews, 37(8), 1838-1859. https://doi.org/10.1016/j.neubiorev. 2013.07.003.

Steele, V. R., Maxwell, A. M., Ross, T. J., Stein, E. A., \& Salmeron, B. J. (2019). Accelerated intermittent theta-burst stimulation as a treatment for cocaine use disorder: A proof-of-concept study. Frontiers in Neuroscience, 13, 1147.

Su, H., Zhong, N., Gan, H., Wang, J., Han, H., Chen, T., et al. (2017). High frequency repetitive transcranial magnetic stimulation of the left dorsolateral prefrontal cortex for methamphetamine use disorders: A randomised clinical trial. Drug and Alcohol Dependence, 175, 84-91. https://doi.org/10.1016/j. drugalcdep.2017.01.037.

Terraneo, A., Leggio, L., Saladini, M., Ermani, M., Bonci, A., \& Gallimberti, L. (2016). Transcranial magnetic stimulation of dorsolateral prefrontal cortex reduces cocaine use: A pilot study. European Neuropsychopharmacology, 26(1), 37-44. https://doi.org/10.1016/j.euroneuro.2015.11.011.

Uhl, G. R., Drgon, T., Johnson, C., Li, C.-Y., Contoreggi, C., Hess, J., et al. (2008). Molecular genetics of addiction and related heritable phenotypes: Genome wide association approaches identify "connectivity constellation" and drug target genes with pleiotropic effects. Annals of the New York Academy of Sciences, 1141, 318. https://doi.org/10.1196/annals.1441.018.

Verdejo-García, A., Bechara, A., Recknor, E. C., \& Perez-Garcia, M. (2006). Executive dysfunction in substance dependent individuals during drug use and abstinence: An examination of the behavioral, cognitive and emotional correlates of addiction. Journal of the International Neuropsychological Society: JINS, 12(3), 405-415. https://doi.org/10.1017/s135 5617706060486.

Vinet, L., \& Zhedanov, A. (2010). A “missing” family of classical orthogonal polynomials. American Journal of Psychiatry, 991. https://doi.org/10.1088/1751-8113/44/8/085201.

Wareham, J. D., \& Potenza, M. N. (2010). Pathological gambling and substance use disorders. The American Journal of Drug and Alcohol Abuse, 36(5), 242-247.

World Health Organization. (2018). ICD-11 for mortality and morbidity statistics (2018). https://icd.who.int/browse11/1-m/en.

Younes, F., Halawi, G., Jabbour, H., El Osta, N., Karam, L., Hajj, A., et al. (2016). Internet addiction and relationships with 
insomnia, anxiety, depression, stress and self-esteem in university students: A cross-sectional designed study. PloS One, 11(9), e0161126. https://doi.org/10.1371/journal.pone. 0161126.

Zhou, Z., Yuan, G., \& Yao, J. (2012). Cognitive biases toward Internet game-related pictures and executive deficits in individuals with an Internet game addiction. PloS One, 7(11). https://doi.org/10.1371/journal.pone.0048961.
Zilverstand, A., Huang, A. S., Alia-Klein, N., \& Goldstein, R. Z. (2018). Neuroimaging impaired response inhibition and salience attribution in human drug addiction: A systematic review. Neuron, 98(5), 886-903. https://doi.org/10.1016/j. neuron.2018.03.048.

Zung, W. W. K. (1971). A rating instrument for anxiety disorders. Psychosomatics, 12(6), 371-379. https://doi.org/10.1016/S00333182(71)71479-0. 